\title{
Academy of Canadian Executive Nurses Annual Report, 2003-04
}

Patricia O'Connor, RN, MSc(A)

President, ACEN, 2003-04

The year 2003-04 was characterized by significant expansion of strategic alliances with key national healthcare organizations and government, successful completion of several patient safety and nursing workload initiatives and preparation for the development of a permanent secretariat in Ottawa for the Academy. These were the particular directions chosen for the Academy at the 2003 annual meeting.

It has been a tremendous honour to represent the members of the Academy by serving as President throughout this past year. The level of commitment and contribution by so many ACEN members towards achieving our objectives is both a hallmark of the Academy and the reason for its successes.

In particular, I wish to thank the members of the Executive Committee Kaaren Neufeld (MB), Past President; Barb McGill (NB), Vice President; Wendy Hill (AB), Secretary-Treasurer; Louise Jones (NF), Communications; and Mary Ferguson-Paré (ON), Archivist - for their leadership and support.
During this past year, the Academy also benefited enormously from the counsel, wisdom and connections of consultant Dr. Mary Ellen Jeans, who worked closely with the Executive Committee in its various endeavours.

I have reported below on ACEN's activities as they relate to the overall goal and objectives of the Academy, based on our strategic directions set in April 2002.

\section{Objective 1. To influence and} participate in setting the directions for healthcare policy and dialogue in \section{Canada}

\subsection{Improving Patient Safety}

One of the Academy's key priorities is ensuring improvements in patient safety.

- ACEN Patient Safety Workgroup: Led the development of several initiatives promoting patient safety since its creation in 2002.

- Canadian Patient Safety Institute (CPSI): In December 2003, two ACEN members (Wendy Nicklin and Patricia Petryshen) were appointed as members 
of the founding Board of this important new body that will provide leadership and coordination to strengthen a culture of safety and quality in the Canadian healthcare system.

- In February 2004, ACEN published the results of a national survey of ACEN members focusing on current safety culture/practices and leadership initiatives in our organizations (CJNL 17: 1). The paper is entitled "Patient Safety Culture and Leadership within Canada's Academic Health Science Centres: Towards the Development of a Collaborative Position Paper."

- In May 2004, ACEN issued a press release: "Response to the Baker-Norton Study on Patient Safety and Adverse Events in Canadian Hospitals.”

- ACEN Safe Practices Inventory: Work began in 2002 and is being completed in fall 2004. It involves the creation of a resource guide of patient safety tools in use in Canadian academic health science centres.

- Other publications on patient safety in 2003-04 by ACEN members: W.

Nicklin, D. Affonso, M. Ferguson-Paré.

- Fall 2004: Publication of a Joint Policy

Statement by ACEN and the Association of Canadian Academic Healthcare Organizations (ACAHO) entitled "Patient Safety Culture and Leadership in Canada's Academic Health Sciences Centres" (Healthcare Quarterly and CJNL).

\subsection{Nursing Workload}

- In summer 2004, ACEN published a Position Statement on Nursing Workload (CJNL) to assist and guide nurses and employers in monitoring the quality of work environments in Canada. It is the Academy's position that quality outcomes, safe patient care and retention of adequate numbers of nurses are significantly influenced by nursing workload and staffing levels.

- Collaborations with the Canadian College of Healthcare Accreditation (CCHSA), the Office of Nursing Policy (ONP), the Canadian Nurses Association (CNA) and the Canadian College of Health Service Executives (CCHSE) have focused on broad issues of quality work environments.

- ACEN has also been a formal partner with nurse researchers who are examining the impact of restructuring on nurse managers and senior nurse leaders.

1.3 Public Health, SARS and Follow-up on Recommendations of CNAC Report

- In December 2003, Kaaren Neufeld (MB) represented ACEN, along with other national nursing organizations, at an invitational Roundtable with the Federal Minster of Health in order to review the implementation date of recommendations from the Canadian Nursing Advisory Committee Report (CNAC).

\subsection{Proposal for Permanent ACEN Secretariat}

In order to support the achievement of the Academy's goals and objectives, the creation of a permanent secretariat in Ottawa is needed, as well as internal restructuring of membership and roles of the Executive Committee and standing committees. 
- In December 2003, ACEN hired a consultant (Dr. Mary Ellen Jeans) to assist us.

- Monthly meetings began in Ottawa between ACEN President, consultant and other national organizations to accelerate ACEN's work.

- September 2004: Obtained membership approval to proceed with permanent secretariat and re-structuring of executive roles and core committees of the Academy.

\section{Objective 2. To contribute to the alignment and advancement of the national nursing practice, education, research and leadership agendas}

\subsection{CHSRF Grants Competition and Nursing Research}

ACEN was sought as a formal partner in the following health services research studies.

- Dr. Heather Laschinger and Carol Wong (ACEN): "A Profile of the Structures and Impact of Nursing Management in Canadian Hospitals" (begun 2003; still in progress. CHSRF Open Grants Competition)

- Dr. Deborah Tregunno: "Leadership to Promote Patient Safety Culture and Learning in Critical Care" (March 2004; CHSRF Open Grants Competition)

ACEN participated in CHSRF Consultation Day on "Future Priorities for Nursing Research.”

\subsection{Consortium for the Advancement of} Nursing Science (Research)

- This is a CNA-led initiative for developing a stronger voice for nursing research in Canada. In October 2004, the third consensus meeting was held with CNA, CNF, CASN, ACEN and the Canadian Association of Nurse Researchers. A formal proposal is now being finalized.

\section{Objective 3. To develop strong strategic coalitions and partnerships with other healthcare leadership groups}

ACEN was invited to participate with several national partners in furthering specific healthcare, research, education or leadership development agendas.

\subsection{Health Canada and Office of Nursing} Policy (ONP)

- Nursing Sector Study: ACEN assisted with publicity and in trying to secure the involvement of Quebec nurses in the study.

- Met with Ian Shuggart to discuss importance of the Office of Nursing Policy and its continuation, and explore upcoming changes within the Canadian Institutes for Health Research.

\subsection{Nursing Leadership Conference (CNA,} ACEN, CHA, CPHA, CASN, CCHSE)

- Planning began in March 2004 for the biennial national conference, to be held in February 2005.

3.3 Canadian Nurses Association (CNA) and Canadian Nurses Foundation (CNF)

- ACEN is proud that Kaaren Neufeld $(\mathrm{MB})$ has again been elected for a two-year term to the CNA Board of Directors, as a representative of the 
Associate and Affiliate Members.

- Provided support to the CNF by advertising its scholarships in CJNL.

\section{4 АСАHO}

- Published Joint Position Paper on Patient Safety.

- Partner with ACAHO and others in proposing to government a national Council on the Future of Academic Health Science Centres in Canada (2003-04).

- Participated with ACAHO in session on content and policy implications of the Health Innovation Canada Proposal.

- Participated with ACAHO CEOs and Vice-Presidents of Research for a fullday conference on research in academic healthcare centres on September 22, 2004.

\subsection{CCHSA}

- February 2004: Explored opportunities for further collaboration, e.g., ACEN's desire to see more national indicators and benchmarks for measuring quality, adverse events and healthier work environments.

- March 2004: Participated in National Consensus Meeting on Work Life Indicators.

\subsection{CCHSE}

- In 2001, ACEN, CCSE and the CAPE (Canadian Association of Physician Executives) sought support from the provincial and territorial deputy ministers of health and Health Canada to conduct an occupational study of health system management and executives in Canada (profiles, background, education, skills).

- Preferred Employer Program: In late Fall 2003, discussions began with various stakeholders interested in raising the standards for human resources management, quality of work life and organizational performance across the healthcare system. CCHSE, ACEN, CNA, ACAHO, ONP, CAPE and CHA began discussions around the development of a broad initiative to institute gold standards of employer practice. In spring 2004, the Office of Nursing Policy provided a small grant matched by partners to develop this proposal further (a Discussion Paper has been developed).

\subsection{Canadian Association of Schools of} Nursing (CASN)

- November 2003: ACEN attended CASN's Annual Board of Directors' Meeting to discuss opportunities for specific collaborations.

- CASN requested involvement by ACEN on a number of dossiers.

\subsection{Canadian Health Services Research} Foundation (CHSRF)

- October 2003: Consulted ACEN (and others) on ways to improve communication.

- March 2004: Attended the CHSRF Invitational Conference focused on Evidence-Based Health Service Research.

- May 2004: Met to discuss partnerships in supporting nursing leadership through the new EXTRA Program. Ontario Best Practice Guidelines Project 
- Louise Jones (NF), Chair of the ACEN Workload Working Group, joined the RNAO BPG advisory committee on Healthy Work Environments.

\subsection{Leaders' Forum for Health Research in} Canada

- Initiated by the Council for Health Research in Canada (CHRC), the Leaders' Forum for Health Research in Canada was held on September 28-29, 2004, in Ottawa. ACEN joined 120 leaders from the health research community to initiate the development of a comprehensive and integrated vision for the health research enterprise of Canada.

\section{Objective 4. To support the develop-} ment of current and emerging executive nurse leaders in Canada

\subsection{Collaboration with Longwoods}

Publishing and Johnson \& Johnson, Inc.

\subsection{ACEN Scholarship}

- This year's scholarship of \$2,500 was awarded to Anne Cooke of Victoria, BC.

4.3 EXTRA Program (Executive Training for Research Application)

- Wendy Nicklin $(\mathrm{ON})$ was selected to be a member of the EXTRA Advisory Board (2003). Dr. Judith Ritchie (McGill) is the other nursing member on the Board.

- Two ACEN members (L. Stevenson and P. O'Connor) were among the 24 senior healthcare leaders chosen in the first cohort. Federal funding supports this program, which is co-sponsored by CHSRF, CNA and CMA.
4.4 Leadership Development Initiatives with Longwoods and Johnson \& Johnson, Inc.

- Explored opportunities for nursing leadership development. A full discussion of these ventures occurred at the 2004 ACEN Annual Business Meeting, along with Anton Hart, owner of Longwoods Publishing.

\section{Objective 5. To provide a forum to discuss and share ideas related to nursing practice, education, research and leadership}

- The website for the Academy was redeveloped this year and launched in winter 2004. Opportunities to expand the site and add important news announcements are now feasible. Web site address is $<\mathrm{http}$ ://www.acen.ca $>$.

- Eleven meetings of the ACEN Executive Committee occurred.

- May 28-29, 2004: Special Meeting of Executive Committee to finalize proposal for secretariat and new internal structure of ACEN (Toronto).

- Four ACEN Update articles were published in CJNL throughout the year.

\section{Objective 6. To support the Editor and Editorial Board in providing the Canadian Journal of Nursing Leadership (CJNL)}

- Longwoods has been publishing our journal (CJNL) for approximately 16 months. This has been an extremely positive venture, and the quality of the journal (scholarly content and appearance) is excellent.

- Circulation is markedly improved, with a large international subscription. 
Bravo to Anton Hart, Dianne

Foster-Kent, Editor-in-Chief

Dorothy Pringle and Mary

Ferguson-Paré (Chairperson of

Editorial Board) for their superb

work.

\section{Closing Remarks}

The Academy continues to consolidate its focus on achieving our primary goals:

- To become a national voice for executive nurse leaders

- To influence and participate in direction setting for healthcare policy

- To contribute to the advancement of the national nursing agenda

- To develop strong strategic partnerships and alliances

Our strength comes from the passion we have for nursing and in knowing that, through effective collaboration with other leaders and government, we can build a better healthcare system and improve the health of our citizens.

Find out how to recruit, retain and combine talents with the best of them.

Join the HealthcareBoard.

Free research, great media and mentoring from the best.

Contact Susan Hale at 4168649667 or shale@longwoods.com

HEALTHCARE BOARD

Longwoods HealthcareBoard would like to thank

MCKESSON

Canada

Empowering Healthcare

For supporting

Breakfast with the Chiefs

It is a measure of their support for learning. Nothing can be more fundamental to the progress of healthcare.

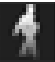

\section{Longwoods HealthcareBoard} would like to thank

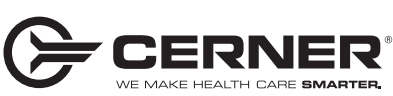

For supporting

Breakfast with the Chiefs

It is a measure of their support for learning. Nothing can be more fundamental to the progress of healthcare.

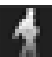

